\title{
Competencias mediáticas para el empoderamiento digital. Estudio de caso de los inmigrantes hispanos en Estados Unidos
}

\author{
Javier Gil-Quintana - Universidad Nacional de Educación a Distancia \\ Sara Osuna-Acedo - Universidad Nacional de Educación a Distancia \\ Carmen Marta-Lazo - Universidad de Zaragoza
}

D) 0000-0003-0326-2535

D 0000-0002-5454-6215

(D) $0000-0002-0004-1094$

Recepción: 31.08.2021 | Aceptado: 16.10.2021

Correspondencia a través de ORCID: Javier Gil-Quintana

iD 0000-0003-0326-2535

Citar: Gil-Quintana, J, Osuna-Acedo, S y Marta-Lazo, C (2021). Competencias mediáticas para el empoderamiento digital. Estudio de caso de los inmigrantes hispanos en Estados Unidos. REIDOCREA, 10(34), 1-11.

Agradecimiento: Este estudio está respaldado por el Grupo en Comunicación e Información Digital (GICID, código S29-17R) y el Grupo de Investigación de Social Media y Educación Mediática Inclusiva y Ubicua (SMEMIU, código 484). GICID pertenece a la Universidad de Zaragoza (España) y está reconocido como grupo de referencia por el Gobierno de Aragón y subvencionado por el Fondo Social Europeo. SMEMIU es un grupo consolidado que pertenece a la Universidad Nacional de Educación a Distancia (UNED) de España.

Área o categoría del conocimiento: Educación Mediática

Resumen: Esta investigación analiza las competencias digitales de los inmigrantes hispanos residentes en Estados Unidos, con el fin de conocer cuáles son sus posibles fortalezas y carencias para alcanzar un verdadero empoderamiento digital ciudadano como grupo. Como técnicas de análisis, se realiza una triangulación metodológica mediante encuestas a los propios inmigrantes, entrevistas en profundidad a observadores privilegiados y focus groups a representantes de diversas disciplinas, todo ello en paralelo con el estudio de los programas radiofónicos de servicio público en la Spanish Public Radio, gestionado por los propios inmigrantes y destinados a su grupo. Se ha concluido que los inmigrantes hispanos residentes en EE.UU. dedican sus consumos en el ciberespacio a un manejo más instrumental del mismo, pero les faltan actitudes críticas para actuar como ciberciudadanía empoderada. El empoderamiento digital de este sector de la población es eficiente para hacerse un hueco en el espacio digital, dar respuesta a sus carencias, sus necesidades y su consolidación como grupo activo e interactivo.

Palabra clave: competencias mediáticas

Media competencies for digital empowerment. Case Study of Hispanic Inmigrants in the United States

Abstract: This research analyzes the digital competencies of Hispanic immigrants residing in the United States, to know what their possible strengths and weaknesses are to achieve a true digital empowerment of citizens as a group. As analysis techniques, a methodological triangulation is carried out through surveys of immigrants themselves, in-depth interviews with privileged observers and focus groups with representatives of various disciplines, all in parallel with the study of public service radio programs in the Spanish Public Radio, managed by the immigrants themselves and destined for their group. It has been concluded that Hispanic immigrants residing in the US dedicate their consumption in cyberspace to a more instrumental management of it, but they lack critical attitudes to act as empowered cybercitizen ship. The digital empowerment of this sector of the population is efficient to gain a foothold in the digital space, to respond to its shortcomings, its needs, and its consolidation as an active and interactive group.

Keyword: media competencies

\section{Introducción}

Los inmigrantes hispanos han buscado formas de empoderamiento que posibiliten la adquisición de herramientas para aumentar su fortaleza, mejorar sus capacidades, acrecentar su potencial (Ramos, 2018) y desarrollar su pensamiento crítico (González Briones y Bernabeu, 2009; Hernández-Pérez, 2018) en Estados Unidos. Los nuevos medios permiten un crecimiento social ilimitado que, a través de un uso colaborativo, innovador y creativo, abre la puerta a la construcción colectiva del conocimiento y a su 
repercusión activa en este segmento social. Hablar de empoderamiento digital en la comunidad de inmigrantes hispanos es descubrir el acceso a la información, capacidad para generar contenidos en plataformas digitales, comunicarse, masificar y compartir sus ideas con otras personas; un planteamiento que se consolida creando redes de conocimiento de forma libre, trabajo e intereses comunes en base a la interacción entre los usuarios.

En la actualidad, estamos sometidos a presiones mediáticas, ante las que nos sentimos vulnerables si no hemos desarrollado una completa competencia digital. Estamos posibilitando que los grupos de poder negocien con gran cantidad de nuestros datos impulsando los sistemas de inteligencia artificial, desde una concepción casi transhumanista (Cortina y Serra, 2015), con lo que "nos acercamos a la creación de un nuevo orden económico donde lo importante es captar información, sin darle ningún valor a las personas" (Lanier, 2018, 1). Dentro de esta espiral del silencio (NoelleNeumann, 1995), donde a los inmigrantes hispanos solo les queda la opción de adaptar sus actitudes y pensamiento a la tendencia de la sociedad estadounidense, se debe apostar por la formación en competencias digitales y mediáticas de la ciudadanía, y más concretamente de este sector de la población. Se trata de una apuesta humanista por la libertad de expresión a través de los nuevos medios, abierta a la interculturalidad, proyectada desde el empoderamiento hispano y luchadora contra el pensamiento único, estereotipos e imaginarios falsos (Valente, 2021). La formación en competencias digitales y mediáticas de los inmigrantes hispanos debe convertirse en una prioridad para el sistema educativo estadounidense, los medios de comunicación, las instituciones y las asociaciones, como líderes intermedios (Osuna-Acedo, Marta-Lazo, y Frau-Meigs, 2018); todo ello acompañado por una reflexión antropológica en torno a quienes somos, de dónde venimos y hacia dónde vamos. Además, es necesario analizar qué medios utilizamos en este camino del aprendizaje para la vida teniendo presentes valores como la responsabilidad, la prudencia, la justicia, el respeto de las libertades y la protección de los grupos más vulnerables. Para el filósofo Lanier (2018), criticar la tecnología es la mejor manera de amarla, puesto que únicamente a través del debate podremos cambiar el rumbo y crear una nueva sociedad digital que sea sostenible, así como menos oscura y alocada.

En función de esta experiencia de investigación en el ámbito de la educación y comunicación, se presenta este artículo, partiendo de los paradigmas clásicos que integran consideraciones teórico-prácticas sobre la comparativa de los valores culturales dependiendo de los diferentes tipos de sociedad (Anderson, 1994; Hofstede, 2003; Inglehart, 1990), con una atención especial al análisis de las relaciones intergrupales en los procesos de inclusión y exclusión social (Bernstein et al., 2010; Deschamps et al., 2005; Triandis et al., 1988; Trompenaars y Hampden-Turner, 1997; Vanhalst et al., 2015) y a la creación de identidad de los inmigrantes, desde un punto de vista multifactorial (Van Dijk, 2005; Wan y Chew, 2013). Asimismo, nos detendremos en el análisis de las representaciones sociales (Leurs et al., 2020; Vecina Marchante, 2012; Wagner, Holtz, y Kashima, 2009) y en la integración para llegar a la multiculturalidad (Ajrouch y Jamal, 2007; Berry, 2011; Guimond y Roebroeck, 2015; Taylor-Gooby, 2013; Unzueta y Binning, 2012). Como base para el análisis de los valores culturales, utilizaremos la tipología de Schwartz y Bardi (2001), establecida en siete valores: conservación, autonomía afectiva, autonomía intelectual, jerarquía, armonía, competencia y compromiso igualitario. 


\section{Objetivos o hipótesis}

Los objetivos en los que se concreta nuestra investigación son los siguientes:

- Objetivo 1: Examinar las diferentes dimensiones competenciales que poseen los inmigrantes hispanos residentes en EE. UU. en su relación con la información recibida por los medios sociales de comunicación.

- Objetivo 2: Contrastar el modo en que los inmigrantes hispanos residentes en EE. UU. se empoderan en los medios sociales de comunicación.

La formulación de estos objetivos responde a un estudio que ha sido proyectado para aproximarse a los fenómenos observables. Asimismo, las hipótesis que presentamos a continuación han sido contrastadas y formuladas utilizando un método hipotéticodeductivo, relacionadas con los objetivos del estudio:

- Hipótesis 1: Los inmigrantes hispanos residentes en EE. UU. valoran que poseen un nivel competencial suficiente de manejo en entornos digitales, pero éste se limita al uso instrumental en su participación en redes sociales, sin valorar adecuadamente una actitud crítica.

- Hipótesis 2: El empoderamiento digital del grupo de inmigrantes hispanos servirá para buscarse un hueco en el espacio digital, dar respuesta a sus carencias y necesidades y consolidarse como grupo activo.

\section{Método}

La propuesta metodológica está basada en un procedimiento mixto. Referente al apartado cuantitativo, el instrumento que se ha utilizado para la recopilación de datos ha sido el cuestionario, cumplimentado por 167 inmigrantes hispanos que viven en los EE. UU. El cuestionario ha sido elaborado siguiendo los criterios de la técnica de la escala de Likert, ofreciendo una afirmación clara al sujeto (Murillo Torrecilla et al., 2014). En relación con la metodología de carácter cualitativo (Barton, Stephens, y Haslett, 2014) se han centrado en el análisis de discurso como referencia fundamental (Iñiguez Rueda, 2011) de 10 entrevistas en profundidad y 3 focus group con la participación de 16 personas. Para la selección de los grupos de discusión que forman parte de la muestra, para su posterior análisis, optamos en primer lugar por coincidir con la nacionalidad de las personas que han participado en la muestra del estudio; posteriormente se seleccionó una variedad atendiendo al criterio de número de miembros, diferentes edades, diverso enfoque, fecha de apertura y diversas categorías profesionales. El perfil de las 167 personas participantes en el estudio cuantitativo es muy variado. El $42,5 \%$ son hombres y $57,5 \%$ mujeres. De ellos, el $17,4 \%$ tiene edades comprendidas entre los 15 y 25 años, el 6,6\% entre 26 y 35, el 38,9 entre 36 y $45,34,7 \%$ entre 46 y 55 , y el $2,4 \%$ entre 55 y 64 años. Se observa también la presencia de diferentes nacionalidades: Cuba 6\%, México 9\%, Guatemala 2,4\%, Haití 0,6\%, España $57,5 \%$, Ecuador $3,6 \%$, Colombia 4,2\%, Honduras 1,8\%, Perú 2,4\%, El Salvador 2,4\%, Venezuela $6 \%$, Nicaragua $2,4 \%$ y México 1,8\%. Por el ámbito de formación académica de las personas participantes en la muestra, observamos un alto porcentaje de un $32,3 \%$ de nivel superior de máster, un $22,2 \%$ de doctorado, un $30,6 \%$ de licenciado y graduado, un 7,8\% educación superior; el 5,4\% tiene una formación académica de bachillerato, $1,2 \%$ educación secundaria y sólo un $0,6 \%$ primaria. Se considera de esta forma un perfil de la muestra muy enriquecedor para poder obtener datos interesantes.

Finalmente, completamos la información del perfil de la muestra haciendo referencia a los ámbitos donde los inmigrantes latinos desarrollan la transferencia profesional de sus conocimientos académicos en EE. UU. Destacamos un $23,4 \%$ de los sujetos que 
trabajan en el entorno educativo y un $13,2 \%$ en la administración y gestión. Resulta llamativo el dato referido al trabajo como investigadores de un $16,8 \%$ de las personas encuestadas y de un $15 \%$ en el ámbito de tecnología y electrónica. El resto de la muestra trabaja en medios de comunicación $(1,2 \%)$, trabajo doméstico $(4,2)$, tecnología y electrónica (15\%), construcción $(5,4 \%)$, salud y deporte $(7,2 \%)$, seguridad $(1,2 \%)$, arte y música $(2,4 \%)$, sector turístico-sociocultural $(1,2 \%)$, estudiante $(3,6 \%)$, desempleado $(4,8)$ y Open-Ended Response $(0,6 \%)$. Por otro lado, la muestra interviniente en las técnicas cualitativas (tablas 1 y 2 ), se distribuye del siguiente modo: en las entrevistas en profundidad, 10 personas, incluido el director de la Spanish Public Radio (SPR), que trabajan con inmigrantes hispanos en EE. UU. y que, en su mayoría, tienen también la condición de inmigrantes hispanos; y en los focus groups, 16 inmigrantes hispanos residentes en EE. UU. El discurso de las entrevistas y de los focus groups fue transcrito de modo literal para la categorización del discurso resultante.

Tabla 1. Perfil de las personas participantes en las entrevistas en profundidad.

\begin{tabular}{|c|c|c|c|}
\hline Nombre & País de origen & Siglas & Perfil \\
\hline Douglas Morgenstern & EE. UU. & E-DM & Profesor Harvard Extension School \\
\hline Daniel Camilo Rojas & Chile & E-DCR & $\begin{array}{l}\text { Investigador y Científico del Massachusetts } \\
\text { Institute of Technology (MIT) }\end{array}$ \\
\hline Pablo Buitrón & Ecuador & E-PB & Profesor Universidad Boston \\
\hline Roberto Ramírez & México & E-RR & Director Spanish Public Radio \\
\hline Javier Laguna & México & E-JL & $\begin{array}{l}\text { Director Universidad Nacional Autónoma de } \\
\text { México (sede en Chicago) }\end{array}$ \\
\hline Erika Erdely & México & E-EE & $\begin{array}{l}\text { Profesora Universidad Nacional Autónoma de } \\
\text { México (sede en Chicago) }\end{array}$ \\
\hline Ramiro Javier Atristain & Bolivia & E-RJ & Profesor en Dominican University \\
\hline Kalman D.Resnik & EE. UU. & E-KR & Abogado Experto en Inmigración \\
\hline Jorge Rennella & Ecuador & E-JR & Periodista Freelancer \\
\hline Jordi Torrent & España & E-JT & $\begin{array}{l}\text { Director y productor de } \\
\text { Civilizaciones de la ONU. }\end{array}$ \\
\hline
\end{tabular}

Tabla 2. Perfil de las personas participantes en los focus groups

\begin{tabular}{lllll}
\hline Número & País de origen & Siglas & Perfil profesional & $\begin{array}{l}\text { Tiempo } \\
\text { EEUU }\end{array}$ \\
\hline 1 & Venezuela & FC1-SO & Empleado & 2 años \\
\hline 2 & Colombia & FC1-CA & Estudiante & 3 años \\
\hline 3 & Chile & FC1-RE & Empleada & 2 años \\
\hline 4 & Argentina & FC1-MA & Estudiante & 3 años \\
\hline 5 & Bolivia & FC1-PE & Estudiante & 3 años \\
\hline 6 & España & FC1-MR & Empleado & 4 años \\
\hline 7 & Panamá & FC1-ME & Empleado & 1 año \\
\hline 8 & España & FC2-SE & Empleado & 5 años \\
\hline 9 & España & FC2-AS & Empleada & 7 años \\
\hline 10 & España & FC2-AM & Desempleada & 2 añoses \\
\hline 11 & España & FC2-CR & Desempleada & 15 años \\
\hline 12 & España & FC2-RE & Empleada & 2 años \\
\hline 13 & Bolivia & FC3-RA & Empleado & 50 años \\
\hline 14 & México & FC3-EE & Empleada & 20 años \\
\hline 15 & México & FC3_RR & Empleado & Autoempleado \\
\hline 16 & Ecuador & FC3-JR & &
\end{tabular}

\section{Resultados}

\section{Categoría 1: Dimensiones competenciales}

Según los datos aportados por la muestra, el 98,8\% de los inmigrantes hispanos en EE. UU. considera su nivel de competencia digital en la búsqueda de información como "bastante" o "mucho", frente a un 1,6\% que lo considera como "aceptable" o "poco". La muestra coincide en que el éxito de estas búsquedas realizadas dependerá de los filtros 
de búsqueda que utilicen. También se pone de manifiesto que el 95,8\% de las personas encuestadas considera que tiene un nivel crítico en la búsqueda de información desde perfiles personales calificado como "bastante" y "mucho", frente a un 4,2\% que lo considera "aceptable". Este proceso crítico de analizar, entender y evaluar para entender y representar el mundo se ha visto reforzada por la valoración de la competencia digital de los inmigrantes hispanos como mucho por un "76\%", bastante por un $13,2 \%$, en contraposición con un $10,8 \%$ que considera que su nivel es "poco" o "aceptable". Por otro lado, se puede apreciar que la competencia digital (X1) es considerada por los inmigrantes hispanos residentes en EE. UU. como relevante para la profesión, pero no tanto para la vida diaria. En la tabla 3 se observa que la competencia digital tiene correlación significativa y positiva con tres variables: búsqueda de información en Internet (X4), uso de filtros (X5) y ser crítico (X6). En las correlaciones se observa que, a mayor competencia digital, mejor actitud de búsqueda de información con criterio en Internet (X4), mejor utilizan filtros para la búsqueda de información (X5) y son más críticos en la búsqueda de información (X6). Las personas que se consideran competentes a nivel digital en su vida profesional buscan mejor información en Internet (X4) y viceversa y utilizan filtros en esta búsqueda (X5). Los más críticos son los que también usan estos filtros (X5) y saben buscar la información en Internet (X4).

Tabla 3. Correlaciones datos cuantitativos respecto a la búsqueda de información en Internet, uso de filtros en la búsqueda y actitud crítica ante la información.

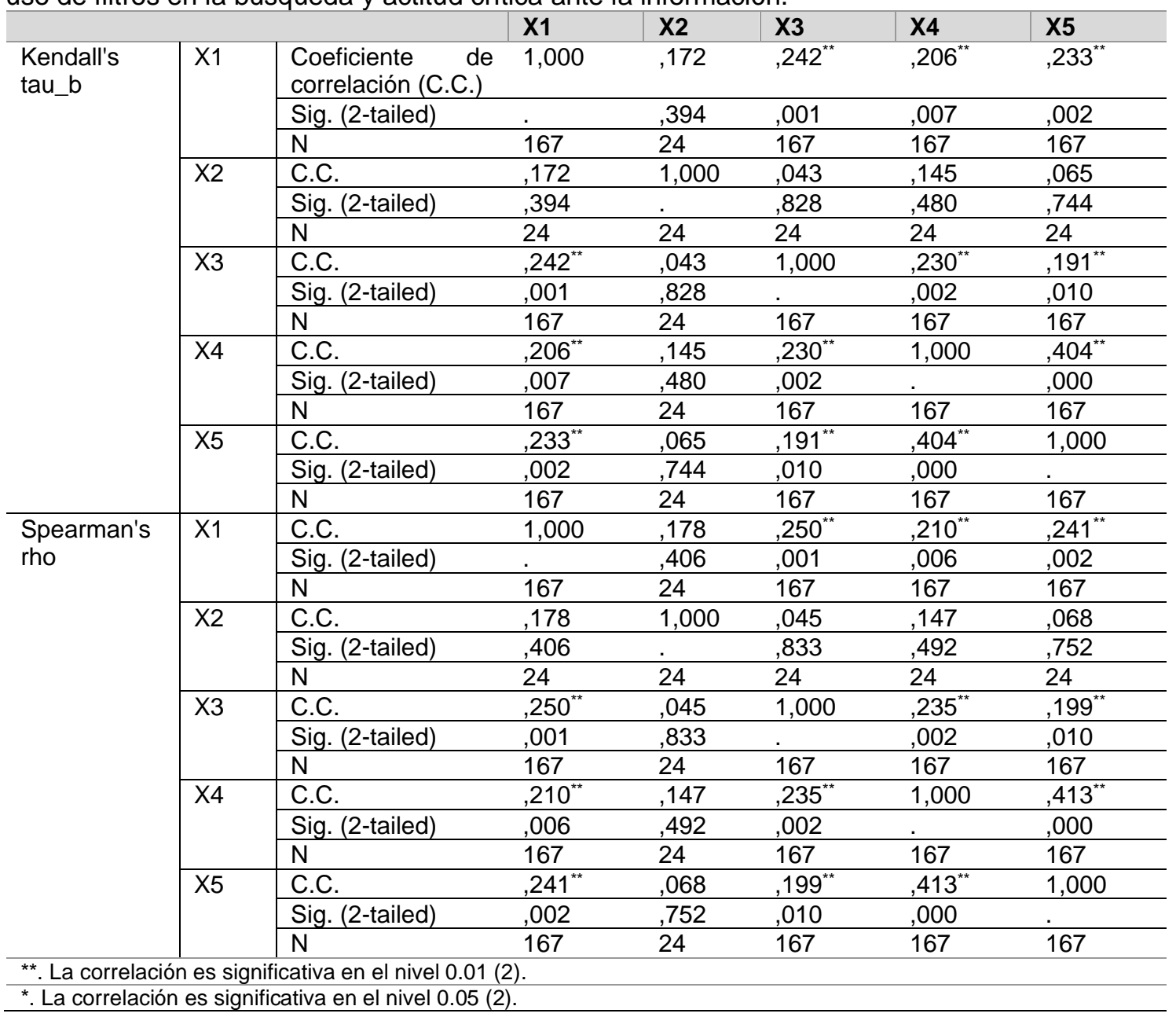

El análisis de los datos cualitativos nos ha permitido extraer significados de datos heterogéneos que no sólo se pueden interpretar de forma cuantificable. De esta forma, en las entrevistas y focus group realizados se comparten impresiones referidas a las ventajas de la búsqueda de información como "pueden tener muchos lugares, muchas 
fuentes, más variadas, pueden aprender cosas, saber más sobre cualquier elemento histórico" (E-DM: 8). Resulta destacable en la investigación que, presentando los inmigrantes hispanos un nivel elevado de competencia digital, búsqueda de información y uso de filtros en el proceso, manifiestan su preocupación por el buen uso, los peligros en el uso de plataformas y una actitud crítica, compartiendo afirmaciones como "evitar que las conversaciones solo se enfoquen en las ramas pequeñas sin tomar en cuenta que, realmente, tener la idea del bosque es importante" (E-PB: 4); "estas plataformas están debilitando sus mentes, en vez de entrar y buscar plataformas que realmente les pueden educar y que les puedan asesorar en ciertas cosas" (E-RR: 7); "¿hasta qué punto las plataformas en sí son un instrumento para la reflexión educativa audiovisual? [...] si no hay un educador que guíe, yo creo que no es tan posible" (E-JT: 8).

\section{Categoría 2: El empoderamiento digital}

Los inmigrantes hispanos residentes en EE. UU. consideran como un asunto prioritario su empoderamiento digital a nivel personal, social y profesional. Respecto a la valoración sobre la relevancia de la competencia digital en la vida profesional el $94 \%$ lo califica como "destacado" concediendo importancia vital al desarrollo de los conocimientos tecnológicos y digitales para su transferencia laboral y ocupación profesional. Los datos presentados en las tablas anteriores quedan reforzados por las cuestiones reflexionadas y debatidas con instrumentos cualitativos. Las personas entrevistadas y participantes en los focus group ponen de manifiesto la necesidad de tener un alto nivel de competencia digital, al estar significativamente relacionada con la vida profesional. Se presentan afirmaciones como "contactar por Facebook, a veces por Skype, a veces hay que contactar el amigo del amigo del amigo que tiene más contacto directo con él, y yo creo que así que se refiere agilidad para saber manejar esas redes" (E-DCR: 4); "tu valor como investigador se basa mucho en que también diseminas en las redes sociales entonces eso es importante" (E-PB: 5); "esta situación de conectarnos con estudiantes en micro para aprender inglés, entonces todos los estudiantes pasan por los sistemas de conversación a distancia con México" (E-JL: 3).

Es interesante, partiendo de la presencia de profesionales de la educación en el perfil de las personas participantes, la importancia que otorgan a la formación y a la labor del educador como agente imprescindible para el desarrollo de la competencia digital, pero desde una línea pedagógica y humanizadora: "la gente que más se motiva es gente que ha tenido problemas de aprendizaje, que sus técnicas actuales de estudio no les están funcionando [...] los educadores quieren encontrar otras técnicas de educar, de mostrar, de enseñar, esa gente es la que se motiva" (E-PB: 4). Esta labor como agente intermedio no queda al margen de su formación continua y actualizada, no sólo en escenarios analógicos sino también en escenarios virtuales e innovadores de aprendizaje "una competencia digital es importante; yo creo deberías como educador, no tanto como investigador, pero como educador, deberías tener la competencia de poder crear estos módulos, que puedas crear un MOOC" (E-PB: 5). Estos espacios se observan, no sólo como entornos para desarrollar la competencia digital, sino también como proyección de: "la creación mediática, una manera de desarrollar los temas de humanidades y de educación cívica, dentro de la educación" (E-JT: 4). Los datos cuantitativos y cualitativos se ven enriquecidos por el análisis también cualitativo de los programas de SPR: "Voces del español", "Alas de amor", "Al mediodía", "Bienestar emocional", "Café, salud y vida", "Chicago voces", "Ciencia y espiritualidad", "Ecuador conmigo desde Chicago", "El consulado a tu lado", "Inspirando la mujer latina", "Liderazgo para negocios Global", "Ponte en forma", "Punto de vista", "SPR contigo", "SPR y tu dinero" y "Un buen día". En estos programas, se pone de manifiesto el interés por colaborar en la vida individual, profesional y social en el empoderamiento digital de los inmigrantes hispanos. SPR incluye música, arte, cultura, ciencia, salud, temas financieros, sociales e información de contenido motivador y educativo. La alfabetización mediática e informacional 
demanda la necesidad de incorporar una actitud crítica con el propósito de crear una mirada analítica que posibilite el entendimiento de aquellos mensajes que no se aprecian claramente.

Finalmente, un aspecto fundamental en la valoración de los inmigrantes hispanos en EE. UU. es la distinción de los sitios seguros y fiables de aquellos donde no es conveniente compartir información personal, grupal o íntima; la fiabilidad presupone que desarrollemos, previamente a la búsqueda o exposición en Internet, conocimiento de las circunstancias de riesgo que se alejan de nuestra confianza. La muestra pone de manifiesto que un $74,9 \%$ considera que distingue sitios seguros y fiables "mucho", un $17,4 \%$ bastante, frente a un $7,2 \%$ como "aceptable" y un 0,6 "poco".

Estos datos se complementan con los aportados a través de los instrumentos cualitativos sobre la actitud crítica, imagen e identidad de los inmigrantes hispanos clave para el empoderamiento en la realidad digital. Las personas entrevistadas ponen de manifiesto que a los inmigrantes hispanos "les interesa más compartir información que confirma su suposición que pensar en las consecuencias de esa publicación para el futuro y con los jóvenes es todavía peor" (E-DCR: 5); "son críticos, pero no tienen la educación suficiente para hacer una crítica adecuada de la información y, lamentablemente, tanto las redes sociales como Internet en general la forma en que se mide la información no es una forma objetiva" (E-PB: 6). Se percibe una preocupación, en un contexto social como el de EE. UU., sobre las fake news y otras mentiras que se proyectan por la red, "la época digital con cada progreso avance con la ética, con la tecnología, llega otro peligro" (E-DM: 11); "ponen los medios sociales una noticia y tú la lees y crees que es real, pero puede ser una mentira o simplemente una distorsión bárbara de la realidad, una verdad a medias" (E-JR: 8); por este motivo "es necesario el control [...] que haya luz verde y luz roja, que haya semáforos [...] es necesario que haya control en las redes" (E-JR: 8). En escenarios como las redes sociales, se proyecta, además de la mentira, "conductas violentas, xenófobas, racistas" (E-RR: 8), preocupando este hecho a las personas entrevistadas y participantes en los grupos de discusión; incluso se manifiestan otras preocupaciones como prevenir toda la actuación de los predadores que pueda haber en Internet y que tratamos a los niños". (E-JL: 9). Esto nos lleva a una necesidad de tener presente la desintoxicación digital, al ser la adicción mediática un hecho preocupante entre los inmigrantes hispanos donde se "crea una especie de necesidad de estar conectado todo el rato, y de no poder estar mirando a las nubes, o viendo cómo crece el césped" (E-JT: 7).

Sin embargo, no podemos olvidar los hechos que convierten a las redes sociales en escenarios para el cambio social, "esto ayuda a conectarse; los movimientos que ha habido, canalizado hasta distintas partes, lo que se llama la primavera árabe, sin los social media hubiese sido más difícil, o más lenta en producirse" (E-JT: 7). En este sentido, no podemos olvidar que "cambia la biología, cambia el ser humano, cambia el mundo, cambia todo; realmente es maravilloso y pavoroso" (E-JR: 8).

\section{Discusión}

En este estudio se han presentado datos relativos al desarrollo del empoderamiento digital. Los inmigrantes hispanos, de diverso género, edad, nivel académico y perfil laboral (Atienza Montero y Romo Calixto, 2020), tienen la convicción de que poseen un alto nivel de competencia digital, valorando el mismo en su vida cotidiana y en su profesión. Estas destrezas y estrategias se ponen de manifiesto en su alta capacidad para buscar información en Internet y en uso de filtros. Esta información es vital en el contexto estadounidense, porque se proyectó así la realidad digital como un "no-lugar" que puede ayudar a desarrollar una mayor concienciación y compromiso para la 
transformación de la realidad social. Este objetivo es posible de conseguir, no sólo con la producción de mensajes como prosumidores (Toffler, 1995), sino también, como emirec (Cloutier, 1973), creando comunidades virtuales al modo de la SPR que potencien el trabajo en grupo, poniéndose al servicio de una casa tan potente como es la valoración de la interculturalidad para el orden social, político y cultural de los EE. UU. La ciudadanía que esté socialmente comprometida debe ser capaz de "sacar partido de las posibilidades que ofrece la red para sensibilizar y comprometer al resto de la ciudadanía en torno a causas humanitarias, sociales o culturales" (Ferrés i Prats 2010, 264).

Los inmigrantes hispanos, aprovechando el Factor Relacional que permiten las TRIC Tecnologías de la Relación, la Información y la Comunicación- (Marta-Lazo, MarfilCarmona, y Hergueta-Covacho, 2016), deben participar en estos espacios digitales comunitarios, donde se puede interactuar de manera libre. Esta implicación lleva consigo riesgos. Los inmigrantes hispanos han manifestado que son críticos en su búsqueda de información y en la creación de perfiles y en el cuidado de la proyección de su imagen personal. También se ha puesto de manifiesto que estos grupos distinguen en entre sitios seguros y fiables. Estos datos de la investigación cuantitativa han sido contradictorios en el análisis de los datos cualitativos, donde manifiesta mucho descuido a la hora de publicar datos personales, fotos y otra información que es privada, convirtiéndose otra gran parte de las personas integrantes en merodeadores o espectadores (Nielsen, 2006).

La selección para el trabajo de campo de SPR, una emisora radio de servicio público en español, gestionada por inmigrantes hispanos y con contenidos elaborados por ellos, nos hace recuperar la figura de los líderes intermedios para la transformación ciudadana, con el fin de extraer un análisis de su visión sobre su identidad como inmigrantes y la imagen que ellos mismos proyectan a otros inmigrantes, a los ciudadanos autóctonos y, en general, a la sociedad. Por estos motivos, es necesario que espacios como las redes sociales o medios de comunicación como SPR sigan trabajando, como líderes intermedios, para conseguir que los inmigrantes hispanos residentes en los EE. UU. tengan "un desarrollado sentido crítico y unos conocimientos científicos que permitan distinguir el saber de la creencia y la veracidad del engaño" (Pérez, 2018). Se trata de un tema interdisciplinar que debe ser abordado desde la alfabetización mediática de una "interiorización de saberes incómodos, con una autonomía y perspectivas indelebles, facilitando además una cimentación personal" (Pedraza Serrano 2018, p.183) convirtiendo a la ciudadanía en usuaria activa, comprometids, inconformistas, personas auténticas. La necesidad de impulsar el pensamiento crítico y el conocimiento de los medios de comunicación, desde la competencia digital y mediática, son pilares básicos para contrarrestar la difusión, entre otras cuestiones, de noticias falsas en la red (Pérez Tornero et al. 2018). Es preciso la difusión del pensamiento crítico, comunicando mejor "por qué la democracia, el Estado de Derecho, la protección de minorías y los derechos fundamentales son clave para todos [...] no sólo protege aquello con lo que estamos de acuerdo (sino también) aquello que es molesto" (Santamaría 2017, p.1). Se consolida con este estudio un análisis de la repercusión de la información/desinformación que recibe la ciudadanía a través de su participación e interacción en la convergencia mediática que hunde sus raíces en la idea del ser humano y con la que se forja desde la identidad infantil (Osuna-Acedo, GilQuintana, y Cantillo Valero, 2018).

La situación de los inmigrantes hispanos en EE. UU. requiere de una revisión profunda basada en los valores humanos derivando en optar por la diversidad como núcleo mismo de todos los procesos socioculturales. Estos valores humanos se ven fortalecidos con el empoderamiento digital que va más allá de la utilización instrumental de los dispositivos electrónicos, o la participación en redes sociales. Según los datos 
analizados en este estudio, hablar de empoderamiento digital de los inmigrantes hispanos es formar una actitud crítica ante la gran cantidad de desinformación a la que estamos expuestos, siendo conscientes de la propia identidad e imagen personal que proyectan en la red (Gil-Quintana y Fernández-Galiano Amorós, 2020). Es necesario desarrollar estrategias de aprendizaje en la búsqueda de información a través de diversos canales con el fin de dar respuesta a sus necesidades y consolidarse como grupo activo en las distintas plataformas digitales. Todo esto encuentra su desarrollo potencial en el Factor Relacional, utilizando los nuevos espacios digitales para comunicarse y colaborar de manera efectiva entre las personas de situación social similar y abriendo las puertas a la interculturalidad y al humanismo digital.

Se hace imprescindible, por tanto, apostar por esta interculturalidad con razones como la necesidad social de aceptación de los inmigrantes, la imposibilidad de frenar los flujos migratorios de diferentes culturas. Se impone a todos los seres humanos el problema de concertar unas relaciones justas entre los estados políticos que forman parte de la aldea global. Este problema se agudiza por los conflictos locales que siguen ensangrentando la humanidad, los riesgos de las tensiones entre las grandes potencias y las desigualdades entre los países en su desarrollo económico y social. Todo ello exige a la sociedad estadounidense un proyecto formativo sociocultural basado en modelos pacíficos de convivencia e igualdad de oportunidades (Santillanes, 2021). Esta intervención debe proyectarse desde las escuelas, las instituciones universitarias, las asociaciones, los planteamientos políticos, los medios de comunicación y las redes sociales. Estas instituciones y plataformas unidas, como líderes intermedios, deben favorecer el desarrollo de procesos comunicativos horizontales, bidireccionales, integradores e inclusivos, basados en la cultura del no odio y en un mensaje integrador.

Como futura línea de investigación, se propone el estudio de los tMOOC como una posible fuente interesante para el aprendizaje de las dimensiones competenciales relacionadas con la actitud crítica, en pro de la ciberciudadanía (Osuna-Acedo, MartaLazo, y Frau-Meigs, 2018). En el caso concreto de los inmigrantes hispanos, se observa la necesidad de crear este tipo de canales para la formación, en los que el Factor Relacional genera un mapa de interacciones y de espacios para compartir sus necesidades, intereses y perspectivas, que es un cauce importante para el empoderamiento digital, en todas las vertientes.

Se trata de recuperar la educación como un derecho para la ciudadanía, lo que implica un planteamiento inclusivo, al ser un derecho de todos que deriva en la igualdad de oportunidades para la incorporación en una sociedad democrática. Como señala el artículo 6 de la Declaración de los Derechos del Hombre y del Ciudadano de 1789, 'todos los ciudadanos son iguales y, por ende, pueden ser admitidos en todas las dignidades, los lugares y los empleos públicos, según su capacidad y sin otra distinción más que la de sus virtudes y sus talentos". En estos tiempos complicados para los inmigrantes hispanos en la sociedad estadounidense no podemos olvidar la advertencia de Dante Alighieri en La Divina Comedia: "Los confines más oscuros del infierno están reservados para aquellos que eligen mantenerse indiferentes en tiempos de crisis moral". La mujer y el hombre deben vivir un compromiso político con una apertura hacia el humanismo humanizado y humanizador a nivel mundial, ser educadores de humanidad. 
Atienza Montero, P. y Romo Calixto, C.E. (2020). El efecto de la población inmigrante sobre el empleo y los salarios de los trabajadores nativos. Una investigación empírica para España, 2009-2018. Estudios de Economía Aplicada, 39(3).

Bernstein, M. J., Donald F. S., Steven G., Young, K. H., y Eric C. (2010). Being "In" With the In-Crowd: The Effects of Social Exclusion and Inclusion Are Enhanced by the Perceived Essentialism of Ingroups and Outgroups. Personality and Social Psychology Bulletin, 36(8), 999-1009.

Berry, J. W. (2011). Integration and Multiculturalism: Ways towards Social Solidarity. Papers on Social Representations, 20, 2.12.21

Capote Pérez, L. J. (2018). Derecho, justicia y pensamiento crítico. Dilemata, (26), 197-205.

Cloutier, J. (1973). La communication audio.scripto-visuelle á I'heure des self-Media. Canada: Les Presses de I'Université de Montréal.

Cortina, A. y Miquel-Ángel, S. (2015). ¿Humanos o posthumanos? Singularidad tecnológica y mejoramiento humano. Barcelona: Fragmenta Editorial.

Deschamps, J. Claude, J. V., Sandra M., Rui CL., y Rosa C. (2005). Intergroup relations, racism and attribution of natural and cultural traits. Psicología Política, 30, 27-39.

Ferrés i Prats, J. (2010). Educomunicación y cultura participativa. En Aparici: Educomunicación: más allá del 2.0, 251-266. Barcelona: Gedisa.

Gil-Quintana, J. y Fernández-Galiano Amorós, M. (2020). Publicaciones, interacción, verdades y mentiras de adolescentes españoles en Instagram. Texto Livre: Linguagem e Tecnologia / Lingüística e Tecnología, 13(1).

González Briones, E. y Bernabeu, N. (2009). La noticia y el reportaje. Madrid: Ministerio de Educación. CIDE.

Guimond, S. y Elodie Roebroeck, M.S. (2015). Les representations du multiculturalisme en France: Decalage singulier entre l'individuel et le collectif. Social Science Information, 54(1), 126.

Hernández-Pérez, T. (2018). Ética y maximalismo digital: necesidad del pensamiento crítico sobre las tecnologías. Anuario Think EPI, 12, 352-355.

Hofstede, G. (2003). Culture; consequences: Comparing values, behaviors, institutions, and organizations across nations, 2nd ed. Thousand Oaks, CA: Sage.

Inglehart, R. (1990). Culture shift in advanced industrial society. Princeton: University Press.

Lanier, J. (2018). Ten arguments for deleting your social media accounts right now. Miami: Random House.

Marta-Lazo, C., Marfil-Carmona, R., y Hergueta-Covacho, E. (2016). Social media application to connectivity learning: The use of Relational Factor in Twitter dialectics. Etic net-Revista Cientifica Electrónica de Educación y Comunicación en la Sociedad del Conocimiento, 2(16), 304-319.
Murillo Torrecilla, F. J., Reyes Hernández Castilla, N. H., y MartínezGarrido, C. (2014). Elaboración y evaluación psicométrica de la escala de actitudes hacia la justicia social en educación (EAJSE). Madrid: Universidad Autónoma de Madrid.

Nielsen, J. (2006). Participation Inequality: Encouraging More Users to Contribute. http://www.nngroup.com/ articles/participationinequality

Noelle-Neumann, E. (1995). La espiral del silencio. Barcelona: Paidós.

Osuna-Acedo, S., Gil-Quintana, J., y Cantillo-Valero, C. (2018). La construcción de la identidad infantil en el Mundo Disney. Revista Latina de Comunicación Social, 73(11), 1284-1306.

Osuna-Acedo, S. Marta-Lazo, C., y Frau-Meigs, D. (2018). De SMOOC a tMOOC, el aprendizaje hacia la transferencia profesional: El proyecto europeo ECO. Comunicar: Revista Científica Iberoamericana de Comunicación y Educación, 55(2), 105-114

Pedraza Serrano, J. R. (2018). El documental ético: recurso procedimental, evaluación y pensamiento crítico. e-CO: Revista digital de educación y formación del profesorado, 15, 179-236.

Pérez Tornero, J. M., Samy Tayie, S. T., y Pulido Rodríguez, C. (2018). ¿Cómo afrontar las noticias falseadas mediante la alfabetización periodística? Estado de la cuestión. Doxa Comunicación, 26, 211-235.

Ramos, J. (2018). El desafío de un inmigrante latino en la era de Trump. México: Grupo Editorial Penguin Random House

Santamaría, L. M. (2017). Impulsar el pensamiento crítico y la alfabetización mediática, claves para protegernos de las noticias falsas. Blog Evolución. Transformación y negocio digital.

Santillanes, N. (2021). Trabaho y salud mental de latinoamericanos en Estados Unidos. Más que una paradoja. México: Universidad Autónoma de México-Instituto de Investigaciones Sociales. Revista Mexicana de Sociología, 83(3), 303.

Schwartz, Shalom H. y Anat Bardi (2001). Value hierarchies across cultures. Talking a similarities perspective. Journal of CrossCultural Psychology, 32(3), 268-290.

Taylor-Gooby, P. (2013). Towards a More Pragmatic MultiCulturalism? How the UK policy community sees the future of ethnic diversity policies. Governance, 27(2), 267-289.

Toffler, A. (1995). La tercera ola. Barcelona: Plaza \& Janes Editores.

Triandis, H. C., Robert Bontempo, M. J. Villareal, M. A., y Nydia L. (1988). Individualism and collectivism: cross-cultural perspectives on self-ingroup relationships. Journal of Personality and Social Psychology, 54 (2), 323-338.

Trompenaars, F. y Hampden-Turner, C. (1997). Riding the waves of culture: Understanding cultural diversity in business. Nueva York: Harper. 
Unzueta, M. M. y Binning, K.R. (2012). Diversity Is in the Eye of the Beholder: How Concern for the In-Group Affects Perceptions of Racial Diversity. Personality and Social Psychology Bulletin, 38(1), 26-38.

Valente, G. (2021). El inmigrante en el cine del siglo XXI: un análisis de la identidad indocumentada y sus esfuerzos por mantener una comunidad unida dentro y fuera de la pantalla. CMC Senior Theses, 2784.

Van Dijk, T. (2005). Ideología y análisis del discurso. Utopía y Praxis Latinoamericana, 29: 9-36.

Vanhalst, J., Soenens, B., Luyckx, K., Van Petegem, S., Weeks, M.S., y Asher, S.R. (2015). Why do the lonely stay lonely? Chronically lonely adolescents' attributions and emotions in situations of social inclusion and exclusion. Journal of Personality and Social Psychology, 109(5), 932-48.

Vecina Marchante, C. (2012). Un estudio sobre representaciones sociales de la inmigración en la prensa y en una revista de barrio. Revista Electrónica de Investigación y Docencia (REID), 32-55.

Wagner, W, Holtz, P., y Kashima, Y. (2009). Construction and Deconstruction of Essence in Representating Social Groups: Identity Projects, Stereotyping, and Racism. Journal for the Theory of Social Behaviour, 39(3), 363-383.

Wan, C. y Yuen-Ga Chew, P. (2013). Cultural knowledge, category label, and social connections: Components of cultural identity in the global, multicultural context. Asian Journal of Social Psychology, 16(4), 247-326. 